\title{
A COMPUTER SYSTEM FOR EFFECTIVE MANAGEMENT OF A MEDICAL LIBRARY NETWORK
}

Richard E. NANCE and W. Kenneth WICKHAM: Computer Science/ Operations Research Center, Institute of Technology, Southern Methodist University, Dallas, Texas, and

Maryann DUGGAN: Systems Analyst, South Central Regional Medical Library Program, Dallas, Texas

TRIPS (TALON Reporting and Information Processing System) is an interactive software system for generating reports to NLM on regional medical library network activity and constitutes a vital part of a network management information system (NEMIS) for the South Central Regional Medical Library Program. Implemented on a PDP-10/SRU 1108 interfaced system, TRIPS accepts paper tape input describing network transactions and generates output statistics on disposition of requests, elapsed time for completing filled requests, time to clear unfilled requests, arrival time distribution of requests by day of month, and various other measures of activity and/or performance. Emphasized in the TRIPS design are flexibility, extensibility, and system integrity. Processing costs, neglecting preparation of input which may be accomplished in several ways, are estimated at $\$ .05$ per transaction, a transaction being the transmittal of a message from one library to another.

\section{INTRODUCTION}

The TALON (Texas, Arkansas, Louisiana, Oklahoma, and New Mexico) Regional Medical Library Program is one of twelve regional programs established by the Medical Library Assistance Act of 1965. The regional programs form an intermediate link in a national biomedical information network with the National Library of Medicine (NLM) at the apex. Unlike 
most of the regional programs that formed around a single library, TALON evolved as a consortium of eleven large medical resource libraries with administrative headquarters in Dallas. A major focus of the TALON program is the maintenance of a document delivery service, created in March 1970 , to enable rapid access to published medical information. TWX units located in ten of the resource libraries and at TALON headquarters in Dallas comprise the major communication channel.

In July 1970 a joint program was initiated to develop a statistical reporting system for the TALON document delivery network. Design and development of the system was done by the Computer Science/Operations Research Center at Southern Methodist University, while training and operational procedures were developed by TALON personnel. Both parties in the effort view the statistical reporting system as a vital first step in providing TALON administrators with a comprehensive network management information system (NEMIS). An overview of this statistical reporting system, designated as TRIPS (TALON Reporting and Information Processing Systems), and its relation to NEMIS is discussed in the following paragraphs. The objectives and design characteristics of NEMIS are stated in (1).

\section{DESIGN REQUIREMENTS}

There were two considerations for rèquirements for a network management information service (NEMIS) for TALON: 1) In what environment would TALON function? 2) What should be the objectives of a network management information service and what part does a statistical reporting system play in its development? The TALON staff and the design team spent an intensive period in joint discussion of these two questions.

\section{TALON Environment}

The TALON document delivery network operates in an expansive geographical area (Figure 1). The decentralized structure of the network enables information transfer between any two resource libraries. In addition TALON headquarters serves as a switching center, by accepting loan requests, locating documents, and relaying requests to holding libraries.

A requirement placed on TALON by NLM is the submission of monthly, quarterly, and annual reports giving statistical data on network activity. These statistics provide details on:

1) requests received by channel used (mail, telephone, TWX, other),

2) disposition of requests (rejected, accepted and filled, accepted and unfilled),

3) response time for filled requests,

4) response time for unfilled requests,

5) most frequent user libraries,

6) requests received from each of the other regions, and

7) non-MEDLARS reference inquiries. 


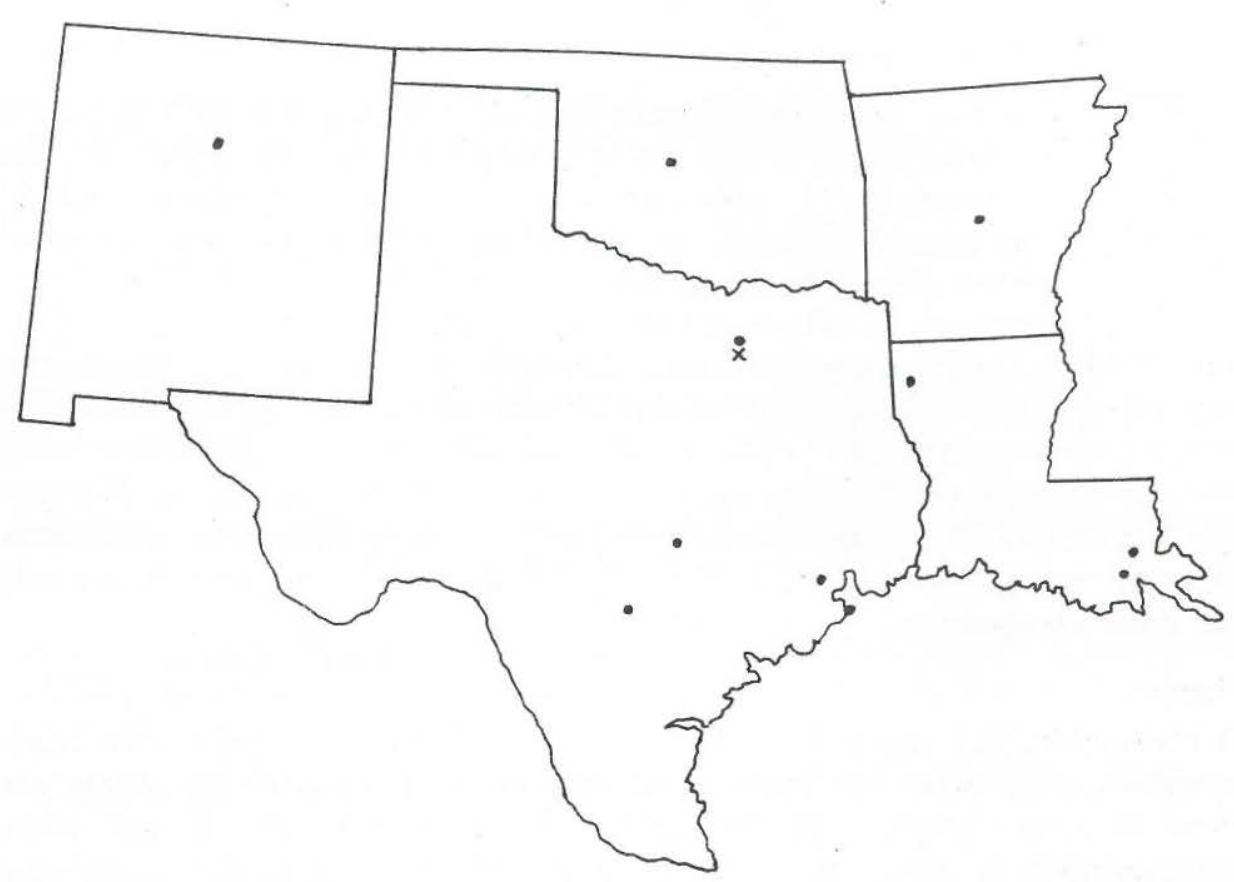

Fig. 1. Location of the Eleven Resource Libraries and TALON Headquarters.

Monthly reports require cumulative statistics on year-to-date performance, and each of the eleven resource libraries and TALON headquarters is required to submit a report on its activity.

\section{Needs and Objectives}

While the immediate need of the TALON network was to develop a system to eliminate manual preparation of NLM reports, an initial decision was made to develop software also capable of assisting TALON management in policy and decision making. Eventual need for a network management information system (NEMIS) being recognized, the TALON reporting and information processing system (TRIPS) was designed as the first step in the creation of NEMIS.

Provision of information in a form suitable for analytical studies of policy and decision making - e.g., the message distribution problem described by Nance (2) - placed some stringent requirements on TRIPS. For instance, the identification of primitive data elements could not be made from report considerations only; an overall decision had to be made that no sub-item of information would ever be required for a data element. In addition the system demanded flexibility and extensibility, since it was to operate in a highly dynamic environment. These characteristics are quite apparent in the design of TRIPS. 


\section{TRIPS DESIGN}

TRIPS is viewed as a system consisting of hardware and software components. The description of this system considers: 1) the input, 2) the software subsystems (set of programs), 3) hardware components, and 4) the output. Emphasis is placed on providing an overview, and no effort is made to give a detailed description.

The environment in which TRIPS is to operate is defined in a single file (FOR25.DAT). This file assigns network parameters, e.g., number of reporting libraries, library codes, and library titles. The file is accessed by subprograms written in FORTRAN IV and DYSTAL (3), the latter being a set of FORTRAN IV subprograms, termed DYSTAL functions, that perform primitive list processing and dynamic storage allocation operations. Because it requires only FORTRAN IV TRIPS can be implemented easily on most computers.

\section{Input}

A transaction log, maintained by each regional library and TALON headquarters, constitutes the basic input to TRIPS. Copies of log sheets are used to create paper tape description of the transactions. If and when compatibility is achieved between standard TWX units and telephone entry to computer systems, the input could be entered directly by each regional library. (This is technically possible at present.) Currently, TALON headquarters is converting the transaction descriptions to machine readable form. Initial data entry under normal circumstances is pictured in Figure 2, which shows the sequence of operations and file accesses in two phases: 1) data entry and 2) report generation. Data entry in turn comprises 1) collecting statistics, 2) diagnosis and verification of input data and 3) backup of original verified input data.

TRIPS is designed to be extremely sensitive to input data. All data is subjected to an error analysis, and a specific file (FOR22.DAT) is used to collect errors detected or diagnosed in the error analysis routine. Only verified data records are transmitted to the statistical accumulation file (FOR20.DAT).

\section{Software Subsystems}

TRIPS comprises seven subsystems or modules. Within each module are several FORTRAN IV subprograms, DYSTAL function and/or PDP-10 systems programs discussed under hardware components in the following section:

NEWY: Run at the beginning of each year, NEWY builds an in-core data structure and transfers it to disk for each resource library in the network. It further creates the original data backup disk file (FOR23.DAT). After disk formatting, RECORD (the accessing and storage module) may be activated to begin accumulating statistics for the new year. 


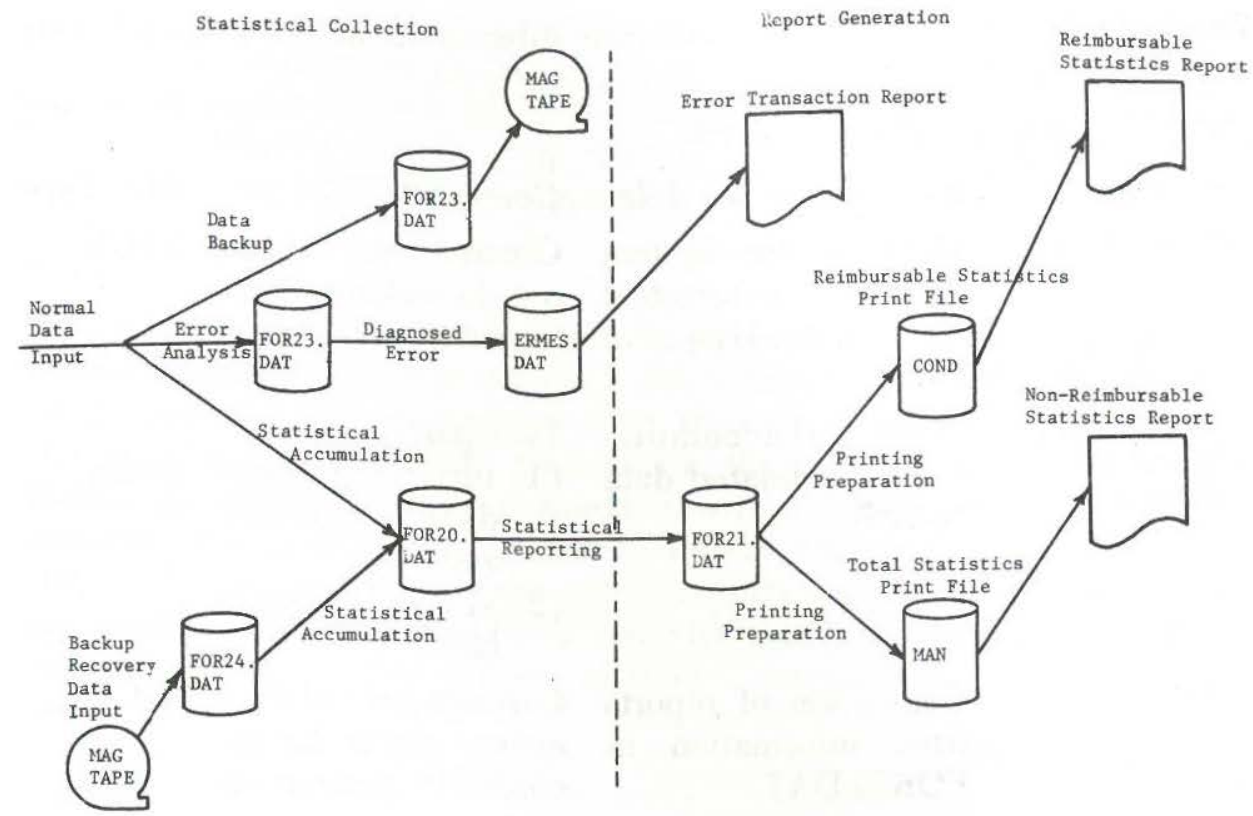

Fig. 2. TRIPS Structure

NEWQ: Run between quarters, NEWQ purges the past quarter statistics for each library and prepares file FOR23.DAT for the next quarter. The report for the quarter must be generated before NEWQ is executed.

NEWM: Run between months, NEWM purges the monthly statistics for each regional library and prepares file FOR23.DAT for the backing up of next month's data.

DUMP1: The utility module causes a DYSTAL dump of the data base.

RECORD: The accessing and storage module RECORD incorporates the error diagnosis on input and the entry of validated data records into file FOR23.DAT. No data record with an indicated error is permitted, and erroneous records are flagged for exception reporting. The error report (ERMES.DAT) may be printed on the teletype or line printer after execution of RECORD.

REPORT: The reporting module REPORT generates all reimbursable statistics on a month-to-date, quarter-to-date, and year-todate basis.

MANAGE: Utilization of TRIPS as a network management tool is afforded by MANAGE, which combines statistics from reimbursable and non-reimbursable transactions to generate a report providing measures of total network activity and performance. 
The primary files used by the software subsystems are described briefly in Table 1.

Table 1. Primary Files in TRIPS

File Name

FOR25.DAT

FOR20.DAT

\section{Function of the File}

Contains the system definition parameters and initialization values.

Statistical accumulation for validated data records.

\section{Comments}

Created from card in- ASCII put to assure proper format.

Two parts:

(1) input translator Binary data structure, and

(2) statistical data base.

FOR21.DAT Generation of reports from information in FOR20.DAT.

Carriage control char- ASCII acters must be included to generate reports.

FOR22.DAT Collects data records diagnosed as in error.

Errors accumulated ASCII in FOR22.DAT are transmitted to ERMES.DAT for output.

FOR23.DAT Enables creation and updating of the backup magnetic tape.

FOR24.DAT Enables recovery read of backup tape.

Each month's vali- ASCII dated records added to tape.

Tape information Binary stored prior to transfer of file information to FOR20.DAT.

ERMES.DAT Serves to output messages on data records diagnosed as in error.
If 6 or less errors oc- ASCII cur ERMES is not created and messages are output to teletype. If more than 6 errors, an estimate of typing time is given to user who has option of printing them on the teletype or in a report form on the line printer. 
A major concern in any management information system is the system integrity. In addition to the diagnosis of input data, TRIPS concatenates sequential copies of disk file FOR23.DAT to provide a magnetic tape backup containing all valid data records for the current year. A failsafe tape, containing all TRIPS programs, is also maintained.

\section{Hardware Components}

Conversion of transaction information to machine readable form is done off line currently. Using a standard TWX with ASCII code, paper tapes are created and spliced together. Fed through a paper tape reader to a PDP-10 (Digital Equipment Company), the input data is submitted to TRIPS. Control of TRIPS is interactive, with the user monitoring program execution from a teletype. All file operations are accomplished using the PDP-10 via the teletype, and the output reports are created on a high-speed line printer. With SMU's PDP-10 and SRU 1108 interface, report generation can be done on line printers at remote terminals to the SRU 1108 as well.

\section{Output}

TRIPS output consists of a report for each library in the network and a composite for the entire network. The report may be limited to reimbursable statistics or include all statistics. Information includes:

1) Errors encountered in the input phase,

2) Number of requests received by channel,

3) Disposition of requests (i.e., rejected, accepted/filled, accepted/ unfilled, etc.),

4) Elapsed time for completing filled requests or clearing unfilled requests,

5) Geographic origin of requests,

6) Titles for which no holdings were located within the region,

7) Types of requesting institutions,

8) Arrival time distribution of requests by day of month,

9) Invoice for reimbursement by TALON,

$10)$ Node/network dependency coefficient as described by (4).

\section{SUMMARY}

TRIPS is now entering its operational phase. Training of personnel at the resource libraries is concluded, and data on transactions are being entered into the system. Input errors have decreased significantly (from fifteen or twenty percent to approximately two percent). TALON personnel are enthusiastic, and needless to say the regional library staffs are happy to see a bothersome, time-consuming manual task eliminated.

In summary, the following characteristics of TRIPS deserve repeating:

1) With its modular construction, it is flexible and extensible. 
2) Implemented in DYSTAL and FORTRAN IV, it should allow installation on most computers without major modifications.

3) Designed to operate in an interactive environment, it can be modified easily to function in a batch processing environment.

4) TRIPS is extremely sensitive to system integrity, providing diagnosis of input data, reporting of errors, magnetic tape backup of data files, and a system failsafe tape.

5 ) Definition of primitive data elements and the structural design of TRIPS enable it to serve as the nucleus of a network management information system (NEMIS) as well as to generate reports required by NLM.

6) Currently accepting paper tape as the input medium, TRIPS could be modified easily to accept punched card input and with more extensive changes could derive the input information during the message transfer among libraries.

Finally, the processing cost of operating TRIPS, neglecting the conversion to paper tape, is estimated to be $\$ .05$ per transaction (a message transfer from one library to another).

Extensive and thorough documentation of TRIPS has been provided. Availability of this documentation is under review by the funding agency.

\section{ACKNOWLEDGMENT}

Work described in this article was done under contract HEW PHS 1 GO4 LM 00785-01, administered by the South Central Regional Medical Library Program of the National Library of Medicine.

The authors express their appreciation to Dr. U. Narayan Bhat and Dr. Donald D. Hendricks for their contributions to this work.

\section{REFERENCES}

1. "NEMIS - A Network Management Information System," Status Report of the South Central Regional Medical Library Program, October 26, 1970.

2. Nance, Richard E.: "An Analytical Model of a Library Network," Journal of the American Society for Information Science, 21: (Jan.-Feb. 1970), 58-66.

3. Sakoda, James M.: DYSTAL - Dynamic Storage Allocation Language Manual, (Providence, R. I.: Brown University, 1965).

4. Duggan, Maryann, "Library Network Analysis and Planning (LIBNAT)," Journal of Library Automation, 2: (1969), 157-175. 